\title{
Variabilidade agronômica entre genótipos comerciais e experimentais de cenoura com ênfase em análise multivariada
}

Agronomic variability between commercial and experimental carrot genotypes with emphasis on multivariate analysis Variabilidad agronómica entre genotipos de zanahoria comercial y experimental con énfasis en el análisis multivariado

Douglas Correa de Souza

ORCID: https://orcid.org/0000-0003-3956-1342 Hortec Tecnologia de Sementes, Brasil E-mail: douglas.souza@hortec.com.br Sebastião Márcio de Azevedo ORCID: https://orcid.org/0000-0002-2992-5379 Universidade Federal de Lavras, Brasil E-mail: sebastiao.azevedo@ufla.br Paulo César Ossani ORCID: https://orcid.org/0000-0002-6617-8085 Universidade Estadual de Maringá, Brasil

E-mail: ossanipc@hotmail.com

Luciano Peixoto de Faria

ORCID: https://orcid.org/0000-0003-2620-4223 Hortec Tecnologia de Sementes, Brasil E-mail: luciano.faria@hortec.com.br

Wagner Adão Aparecido Marques ORCID: https://orcid.org/0000-0002-0142-0635 Hortec Tecnologia de Sementes, Brasil E-mail: wagner.marques@hortec.com.br

Gustavo Akira Narita

ORCID: https://orcid.org/0000-0001-7294-1991 Hortec Tecnologia de Sementes, Brasil E-mail: gustavo.narita@hortec.com.br

\section{Resumo}

A cenoura está entre as cinco hortaliças mais cultivadas no Brasil, por isso a busca por cultivares comerciais é um grande desafio em programas de melhoramento genético, pois além de produtivas, essas raízes tuberosas devem apresentar características de interesse fundamentais para que o produtor consiga aceitação do produto no mercado consumidor. Diante disso, objetivou-se com esse trabalho avaliar o nível de similaridade entre genótipos de cenoura de verão, e estabelecer as relações entre as variáveis, por meio de caracteres agronômicos e morfológicos da planta e das raízes tuberosas, com o propósito de diagnosticar possíveis agrupamentos em relação às variáveis analisadas, utilizando as técnicas de análises multivariadas e univariada. O ensaio foi conduzido em delineamento em blocos casualizados com três repetições, avaliando 11 genótipos de cenoura de verão, sendo sete genótipos comerciais disponíveis no mercado (SV1099DT, Nativa, EX 4098, AGR-123, AGR-125, Juliana e Brazlândia) e quatro híbridos experimentais. Foram avaliadas 15 características agronômicas das plantas e das raízes tuberosas. Diante dos resultados a técnica de análise de múltiplos fatores foi capaz e estabelecer as similaridades entre os genótipos estudados, mostrando alta similaridade entre os genótipos comerciais presentes no mercado e os híbridos experimentais. A análise de fatorial exploratória estabeleceu as correlações das variáveis, e foi capaz de projetar uma separação entre os genótipos comerciais e experimentais. As especificidades apresentadas pelos genótipos experimentais foram explicadas no teste de média Scott-Knott.

Palavras-chave: Daucus carota L.; Melhoramento genético; Similaridade; Análise de múltiplos fatores.

\footnotetext{
Abstract

Carrots are among the five most cultivated vegetables in Brazil, which is why the search for commercial cultivars is a great challenge in breeding programs, because, in addition to being productive, these tuberous roots must present characteristics of fundamental interest for the producer to achieve acceptance of the product in the consumer market. Therefore, the objective of this work was to evaluate the level of similarity between summer carrot genotypes, and to
} 
establish the relationships between the variables, through agronomic and morphological characters of the plant and tuberous roots, in order to diagnose possible groupings in relation to the analyzed variables, using the techniques of multivariate and univariate analysis. The trial was conducted in a randomized block design with three replications, evaluating 11 summer carrot genotypes, with seven commercial genotypes available on the market (SV1099DT, Nativa, EX 4098, AGR-123, AGR-125, Juliana and Brazlândia) and four experimental hybrids. Fifteen agronomic characteristics of plants and tuberous roots were evaluated. Given the results, the multiple factor analysis technique was able to establish the similarities between the genotypes studied, showing high similarity between commercial genotypes present in the market and experimental hybrids. Exploratory factor analysis established the correlations of the variables and was able to project a separation between commercial and experimental genotypes. The specificities presented by the experimental genotypes were explained in the Scott-Knott mean test.

Keywords: Daucus carota L.; Genetic breeding; Similarity; Multiple factor analysis.

\section{Resumen}

Las zanahorias se encuentran entre las cinco hortalizas más cultivadas en Brasil, por lo que la búsqueda de cultivares comerciales es un gran desafío en los programas de mejoramiento, pues además de ser productivas, estas raíces tuberosas deben presentar características de interés fundamental para que el productor logre aceptación del producto en el mercado de consumo. Por tanto, el objetivo de este trabajo fue evaluar el nivel de similitud entre genotipos de zanahoria de verano, y establecer las relaciones entre las variables, a través de caracteres agronómicos y morfológicos de la planta y raíces tuberosas, con el fin de diagnosticar posibles agrupaciones en relación con las variables analizadas, utilizando las técnicas de análisis multivariado y univariado. El ensayo se realizó en un diseño de bloques al azar con tres repeticiones, evaluando 11 genotipos de zanahoria de verano, con siete genotipos comerciales disponibles en el mercado (SV1099DT, Nativa, EX 4098, AGR-123, AGR-125, Juliana y Brazlândia) y cuatro experimentales. híbridos. Se evaluaron quince características agronómicas de las plantas y raíces tuberosas. Dados los resultados, la técnica de análisis de factores múltiples logró establecer las similitudes entre los genotipos estudiados, mostrando una alta similitud entre los genotipos comerciales presentes en el mercado y los híbridos experimentales. El análisis factorial exploratorio estableció las correlaciones de las variables y pudo proyectar una separación entre genotipos comerciales y experimentales. Las especificidades presentadas por los genotipos experimentales se explicaron en la prueba de medias de Scott-Knott.

Palabras clave: Daucus carota L.; Mejoramiento genético; Similitud, Análisis de factores múltiples.

\section{Introdução}

A cenoura (Daucus carota L.) é originária da Ásia Central, pertencente à família Apiaceae, sendo uma das espécies mais valiosas do grupo das raízes tuberosas. O consumo mundial per capita é de aproximadamente 5,3 kg por ano, podendo ser utilizada como salada, sucos, guisados e como guarnição (FAOSTAT, 2017). Além do sabor agradável é rica em compostos fenólicos, carotenoides, vitaminas, minerais e fibra alimentar (Hiranvarachat \& Devahastin, 2014).

No Brasil, a cultura é cultivada em todo território nacional e apresenta grande relevância social e econômica, sendo o cultivo de verão considerado o mais crítico, ocasionando muitas vezes falta do produto no mercado, com consequente elevação de preços. Durante a primavera e verão os produtores de cenoura tem utilizado genótipos nacionais, como Brasília, Alvorada, Carandaí, dentre outros materiais (Oliveira, et al., 2005).

Diante do exposto, torna-se necessário o desenvolvimento de novos genótipos de cenoura de verão, por isso, empresas públicas e privadas de pesquisa não têm medido esforços em programas de melhoramento genético com a cultura. Nesses programas o estudo da variabilidade genética é considerado uma etapa essencial, uma vez que cruzamentos envolvendo genitores geneticamente divergentes podem induzir maiores efeitos heteróticos e variabilidade nas gerações segregantes, permitindo a construção de populações com uma base genética mais ampla e proporcionando maiores ganhos genéticos com os ciclos de seleção (Cecon, 2008; Cruz, et al., 2012).

A variabilidade genética pode ser observada por meio de estudos morfológicos (quantitativos e qualitativos), assim como da utilização de técnicas moleculares. Entretanto, pesquisas a partir de caracteres agronômicos são os mais utilizados por serem mais acessíveis e contributivos, porém, tem-se necessidade de repetibilidade, por serem descritores com maior influência do fator ambiental (Buratto, et al., 2016).

Várias são as ferramentas que estão sendo utilizadas atualmente para essa finalidade, como a análise de componentes 
principais (PCA), um método multivariado utilizado para determinar a variabilidade genética em populações que permite identificar os caracteres que explicam grande parte da variabilidade existente (Souza, et al., 2019). A PCA permite inferir quais caracteres representam a maior fração da variabilidade encontrada, pois possibilita a retirada daqueles caracteres que apresentam poucas contribuições para a divergência genética da população estudada e reduz o número de caracteres a serem analisados (Cruz, et al., 2012).

Assim, objetivou-se com esse trabalho avaliar o nível de similaridade entre genótipos de cenoura de verão, e estabelecer as relações entre as variáveis, por meio de caracteres agronômicos e morfológicos da planta e das raízes tuberosas, com o propósito de diagnosticar possíveis agrupamentos em relação às variáveis analisadas, utilizando as técnicas de análises multivariadas e univariada.

\section{Materiais e Métodos}

\subsection{Descrição dos dados}

O experimento foi conduzido em delineamento de blocos casualizados (DBC) com três repetições. Foram avaliados 11 genótipos de cenoura de verão, sendo sete genótipos comerciais disponíveis no mercado (SV1099DT [500], Nativa [501], EX 4098 [502], AGR-123 [503], AGR-125 [504], Juliana [505] e Brazlândia [506]) e quatro híbridos experimentais (507, 508, 509 e 510) pertencentes ao programa de melhoramento genético da empresa Hortec Tecnologia de Sementes.

As parcelas experimentais apresentaram $4 \mathrm{~m}^{2}$, com dimensão de $1 \mathrm{~m}$ de largura por $4 \mathrm{~m}$ de comprimento, totalizando uma área total de $132 \mathrm{~m}^{2}$. Sendo conduzido em campo aberto na Estação Experimental da Hortec Tecnologia de Sementes, em

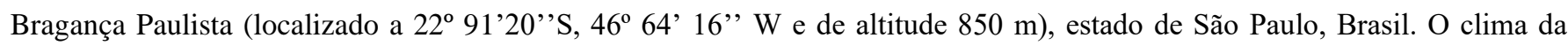
região de acordo com a classificação climática de Koppen é Cfa (subtropical) com temperatura média de $19,6{ }^{\circ} \mathrm{C}$ e pluviosidade média anual de $1.432 \mathrm{~mm}$ (Álvaro, et al., 2013).

O experimento foi semeado em novembro de 2020, e 30 dias após a semeadura (DAS) foi realizado o desbaste de modo que o espaçamento entre plantas foi de dois $\mathrm{cm}$ e entre linhas de $20 \mathrm{~cm}$. Os tratos culturais como adubações de plantio e cobertura, assim como as aplicações de inseticidas, fungicidas e herbicidas foram realizadas de acordo com a necessidade da cultura, seguindo as recomendações de Nick \& Borém (2016). As colheitas foram realizadas aos 95 DAS.

Durante a condução do experimento foram avaliados os caracteres da planta (incidência de queima de folhas, vigor de planta e porcentagem de pendoamento), caracteres produtivos da cultura (produtividade em ton ha ${ }^{-1}$ e número de raízes tuberosas), porcentagem da classificação comercial do comprimento das cenouras (G: $\geq 22 \mathrm{~cm} \mathrm{a}<26 \mathrm{~cm} ; 2 \mathrm{~A}$ : $\geq 15 \mathrm{~cm} \mathrm{e}<22$ $\mathrm{cm} ; 1 \mathrm{~A}: \geq 10 \mathrm{~cm} \mathrm{e}<15 \mathrm{~cm}$; e descarte: $\leq 10 \mathrm{~cm}, \geq 26 \mathrm{~cm}$ e cenouras defeituosas) e as características das raízes tuberosas (cor externa, cor do córtex, cor do núcleo central, formato, presença de raízes secundárias e ombro verde), sendo as características avaliadas por notas mensuradas por dois avaliadores utilizando a escala de notas apresentada na Tabela 1. 
Tabela 1. Notas estabelecidas de 1 a 5 para as diferentes características de plantas e raízes tuberosas da cenoura de verão.

\begin{tabular}{|c|c|}
\hline Características & Notas \\
\hline $\begin{array}{l}\text { IQF: Incidência de queima } \\
\text { de folhas }\end{array}$ & 1: muito ruim; 2: ruim; 3: razoável; 4: bom e 5: muito bom. \\
\hline VP: Vigor de planta & 1: muito ruim; 2: ruim; 3: razoável; 4: bom e 5: muito bom. \\
\hline CE: Cor externa & 1: amarelado; 2: laranja claro; 3: laranja médio; 4: laranja intenso e 5: laranja escuro. \\
\hline CC: Cor do córtex & 1: amarelado; 2: laranja claro; 3: laranja médio; 4: laranja intenso e 5: laranja escuro. \\
\hline CNC: Cor núcleo central & 1: amarelado; 2: laranja claro; 3: laranja médio; 4: laranja intenso e 5: laranja escuro. \\
\hline Forma: Formato da cenoura & 1: muito ruim; 2: ruim; 3: razoável; 4: bom e 5: muito bom. \\
\hline $\begin{array}{l}\text { PR: Presença de raízes } \\
\text { secundárias }\end{array}$ & 1: Excesso; 2:Algumas 3: razoável; 4: poucas e 5: ausente. \\
\hline $\begin{array}{l}\text { OV: Presença de ombro } \\
\text { verde }\end{array}$ & 1: Excesso; 2:Algumas 3: razoável; 4: poucas e 5: ausente. \\
\hline
\end{tabular}

Fonte: Autores.

De acordo com as características avaliadas em cada genótipo de cenouras de verão foi realizada a análise de múltiplos fatores (MFA), análise fatorial exploratória (FA) usando o método do fator principal, sem rotação, sendo o número de fatores escolhidos com base na proporção explicada pelos autovalores e as médias foram comparadas pelo teste de Scott-Knott (1974) ao nível de 5\% de significância, os resultados das análises MFA e FA foram obtidos por meio do desenvolvimento de scripts no software R (R CORE TEAM, 2021) para uso público por meio do pacote MVar versão 2.1.7 (Ossani \& Cirillo, 2021) e ExpDes versão 1.2.1 (Ferreira, et al., 2021) foi utilizado para o teste Scott-Knott.

\subsection{Métodos estatísticas}

Há muitas técnicas multivariadas para analisar dados, mas a análise de múltiplos fatores (MFA) se caracteriza por permitir analisar grupos de variáveis com tamanhos diferentes e de natureza distintas, que podem ser quantitativas, categóricas, de frequência ou mistas, definidos no mesmo conjunto de observações (Escofier \& Pagès, 2008). Deste modo, este método pode ser usado para analisar estudos em que se identifica vários grupos de variáveis, ou para os estudos em que as mesmas perguntas são feitas em intervalos de tempo diversos (Abdi, et al, 2013).

A técnica MFA utiliza a estrutura da variância e covariância de um vetor aleatório, composto de $p$-variáveis aleatórias por meio da construção de combinações lineares das variáveis originais pertencentes a cada grupo de variáveis, as quais são balanceadas conforme a natureza das variáveis em cada grupo, com isso obtendo-se resultados utilizados na avaliação de similaridade entre os grupos.

Segundo Rencher (2012) a análise fatorial exploratória tem por objetivo analisar os padrões de correlações existentes entre as variáveis e utiliza esses padrões de correlações para agrupar suas variáveis em fatores, os quais são variáveis não observadas que se pretende medir a partir das variáveis observadas.

A análise fatorial exploratória utiliza-se de cargas fatoriais que estabelecem as correlações entre as variáveis originais e os fatores encontrados, de modo que para valores em modulo entre $[0 ; 0,3)$ será dito como fraca carga fatorial, $[0,3 ; 0,5)$ 
baixa carga fatorial, $[0,5 ; 0,7)$ moderada carga fatorial, $[0,7 ; 0,9)$ alta carga fatorial e $[0,9 ; 1,0)$ forte carga fatorial.

\section{Resultados e Discussão}

\subsection{Multiple factor analysis}

Os resultados descritos na Tabela 2 evidenciam que o estudo das associações entre as variáveis, analisadas simultaneamente, poderá ser explicada em um gráfico bidimensional, uma vez que $\lambda_{i}$ a proporção da variação explicada nos dois componentes é igual a $100 \%$.

Tabela 2. Proporção da variação amostral explicada pelos nos componentes.

\begin{tabular}{cccc}
\hline Componentes & $\lambda_{i}$ & \% da variação amostral & \% da variação amostral acumulada \\
\hline 1 & 9,8252 & 58,05 & 58,05 \\
2 & 7,0991 & 41,95 & 100 \\
\hline
\end{tabular}

Fonte: Autores.

Em se tratando da inércia parcial de cada genótipo, interpretada como a quantidade de variação restituída em cada componente inerente a análise global, os resultados descritos na Tabela 3 afirmam que a primeira componente, apresenta uma forte similaridade entre os genótipos 500,503, 504, 506, 508, 509 e 510, enquanto os demais genótipos apresentaram alta similaridade entre si com destaque para os genótipos 502 e 505. Embora todos os genótipos foram explicados na primeira componente, ressalta-se na segunda componente que houve forte similaridade entre os genótipos 501 e 502, alta similaridade entre os genótipos 503, 505 e 510, moderada similaridade entre os genótipos 500 e 506, e houve baixa similaridade entre os genótipos 504, 507, 508 e 509.

Das interações entres as componentes podemos destacar os genótipos 501, 503, 505 e 510 por apresentarem altas e/ou fortes similaridades, enquanto os genótipos comerciais 500, 502 e 506 apresentam moderada similaridade em ambas as componentes. Ou seja, $85,70 \%$ dos genótipos comerciais estudados apresentam similaridade moderada (42,85\%) e alta $(42,85 \%)$ entre si. A partir das inércias obtidas em cada grupo na Tabela 3, visando uma melhor interpretação, o gráfico das inércias foi gerado e apresentado na Figura 1.

Tabela 3. Valores referentes às inércias computadas nos eixos para cada tratamento.

\begin{tabular}{ccccccccccccc}
\hline \multirow{2}{*}{ Comp. } & \multicolumn{10}{c}{ Código } \\
\cline { 2 - 16 } & 500 & 501 & 502 & 503 & 504 & 505 & 506 & 507 & 508 & 509 & 510 & Inércias \\
\hline 1 & 0,997 & 0,863 & 0,689 & 0,951 & 0,924 & 0,716 & 0,914 & 0,876 & 0,999 & 0,897 & 0,999 & 9,8252 \\
2 & 0,545 & 0,981 & 0,910 & 0,879 & 0,393 & 0,821 & 0,613 & 0,433 & 0,326 & 0,441 & 0,757 & 7,0991 \\
\hline
\end{tabular}

Coeficientes de similaridade (CS): fraca similaridade entre $0,0 \leq \mathrm{CS}<0,3$; baixa $0,3 \leq \mathrm{CS}<0,5$; moderada $0,5 \leq \mathrm{CS}<0,7$; alta $0,7 \leq \mathrm{CS}<0,9$ e forte $0,9 \leq \mathrm{CS} \leq 1,0$. Fonte: Autores. 
Figura 1. Inércias dos grupos provenientes da avaliação de sete genótipos comerciais e quatro híbridos experimentais de cenoura.

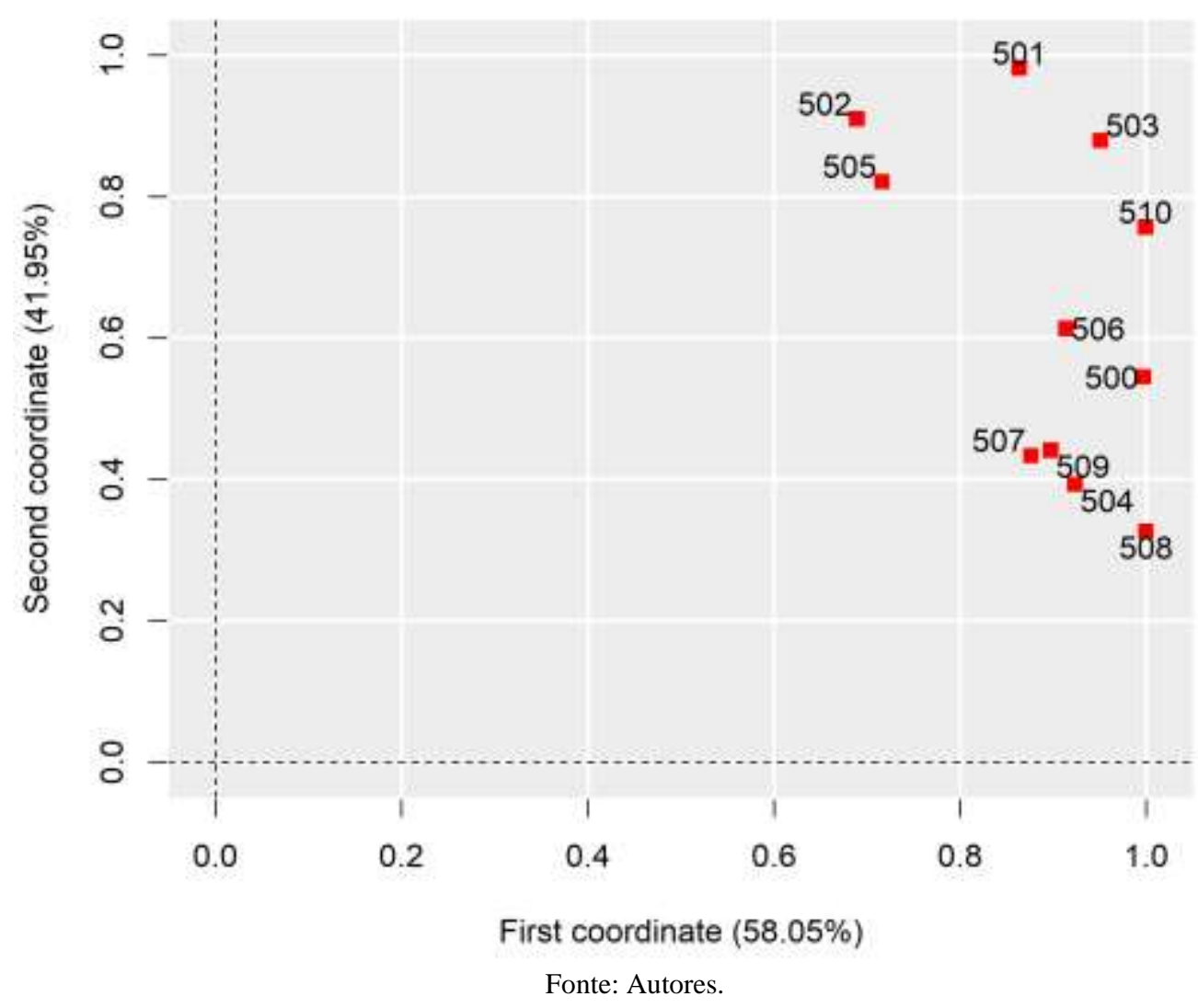

\subsection{Análise fatorial exploratória}

Nas análises o número de fatores foi escolhido com base na proporção explicada pelos autovalores, assim utilizou-se quatro fatores, que somados explicam 77,32\% da variância amostral, tendo os resultados apresentados na Tabela 4.

Ao considerar os resultados, a variável que menos foi explicada pelo modelo fatorial foi porcentagem de pendoamento, enquanto a melhor explicação foi dada para a classificação de raízes 1A, esses resultados podem ser facilmente verificados tomando-se as porcentagens da variância que é devida à comunalidade de cada variável, considerando-se o modelo fatorial ajustado.

As explicações das variâncias atribuídas nos dois primeiros fatores são respectivamente de 93,13\% e 3,68\%, representando a variação total de 96,81\%, a Figura 2, mostra a magnitude da variabilidade de todos os fatores encontrados.

A Tabela 4 mostra as correlações das variáveis analisadas com os fatores, estabelecendo em modulo as cargas fatoriais fraca, baixa, moderada e alta nos limites estabelecidos na seção 2.2, tendo os valores positivos e negativos estabelecendo as relações inversas. Essas relações podem ser visualizadas na Figura 2. 
Tabela 4. Resultados da análise fatorial exploratória para os caracteres agronômicos e morfológicos da planta e das raízes tuberosas.

\begin{tabular}{|c|c|c|c|c|c|c|}
\hline \multirow{2}{*}{ Variáveis } & \multicolumn{4}{|c|}{ Fatores } & \multirow{2}{*}{ Comunalidade } & \multirow{2}{*}{$\begin{array}{l}\text { Variância } \\
\text { específica }\end{array}$} \\
\hline & 1 & 2 & 3 & 4 & & \\
\hline Incidência de queima de folhas & $-0,164$ & 0,641 & 0,325 & $-0,154$ & 0,567 & 0,433 \\
\hline Número de raízes tuberosas/ha & $-0,570$ & 0,214 & 0,089 & 0,171 & 0,408 & 0,592 \\
\hline Produtividade de cenoura/ha & $-0,661$ & 0,399 & 0,238 & 0,199 & 0,693 & 0,307 \\
\hline Porcentagem de pendoamento & 0,372 & $-0,088$ & $-0,090$ & $-0,081$ & 0,161 & 0,839 \\
\hline Classificação: G & $-0,336$ & 0,593 & $-0,420$ & $-0,073$ & 0,646 & 0,354 \\
\hline Classificação: 2A & $-0,589$ & $-0,087$ & 0,351 & 0,594 & 0,830 & 0,170 \\
\hline Classificação: 1A & $-0,417$ & 0,030 & $-0,708$ & $-0,440$ & 0,869 & 0,131 \\
\hline Classificação: Descarte & 0,806 & $-0,191$ & 0,230 & $-0,235$ & 0,794 & 0,206 \\
\hline Nota para cor externa & $-0,631$ & $-0,562$ & $-0,145$ & 0,175 & 0,766 & 0,234 \\
\hline Nota para cor do córtex & $-0,842$ & $-0,329$ & $-0,091$ & $-0,154$ & 0,849 & 0,151 \\
\hline Nota para cor do núcleo central & $-0,848$ & $-0,190$ & $-0,035$ & $-0,164$ & 0,783 & 0,217 \\
\hline Nota para formato & $-0,647$ & 0,132 & 0,344 & $-0,537$ & 0,843 & 0,157 \\
\hline Nota para raízes secundárias & $-0,576$ & $-0,099$ & 0,435 & $-0,293$ & 0,617 & 0,383 \\
\hline Nota para ombro verde & $-0,325$ & 0,106 & $-0,513$ & 0,376 & 0,521 & 0,479 \\
\hline Nota para vigor de planta & 0,149 & 0,724 & $-0,070$ & 0,079 & 0,558 & 0,442 \\
\hline Variância & 4,915 & 2,043 & 1,638 & 1,309 & 9,905 & - \\
\hline \% variância & 38,37 & 15,94 & 12,79 & 10,22 & 77,32 & - \\
\hline
\end{tabular}

Fonte: Autores.

Figura 2. Correlação para os caracteres agronômicos e morfológicos da planta e das raízes tuberosas nos dois primeiros fatores.



Fonte: Autores. 
A Figura 3 mostra a dispersão dos pontos que representam os genótipos estudados, caracterizando grande dispersão, e mostrando uma separação entre os genótipos comerciais e experimentais, tendo este último formando um agrupamento no primeiro e segundo quadrantes, demonstrando a especificidade destes, embora apresentando alta similaridade no método MFA. Tais especificidades foram capitadas no teste de média apresentado na seção seguinte.

Figura 3. Projeção de sete genótipos comerciais e quatro híbridos experimentais nos dois primeiros fatores.

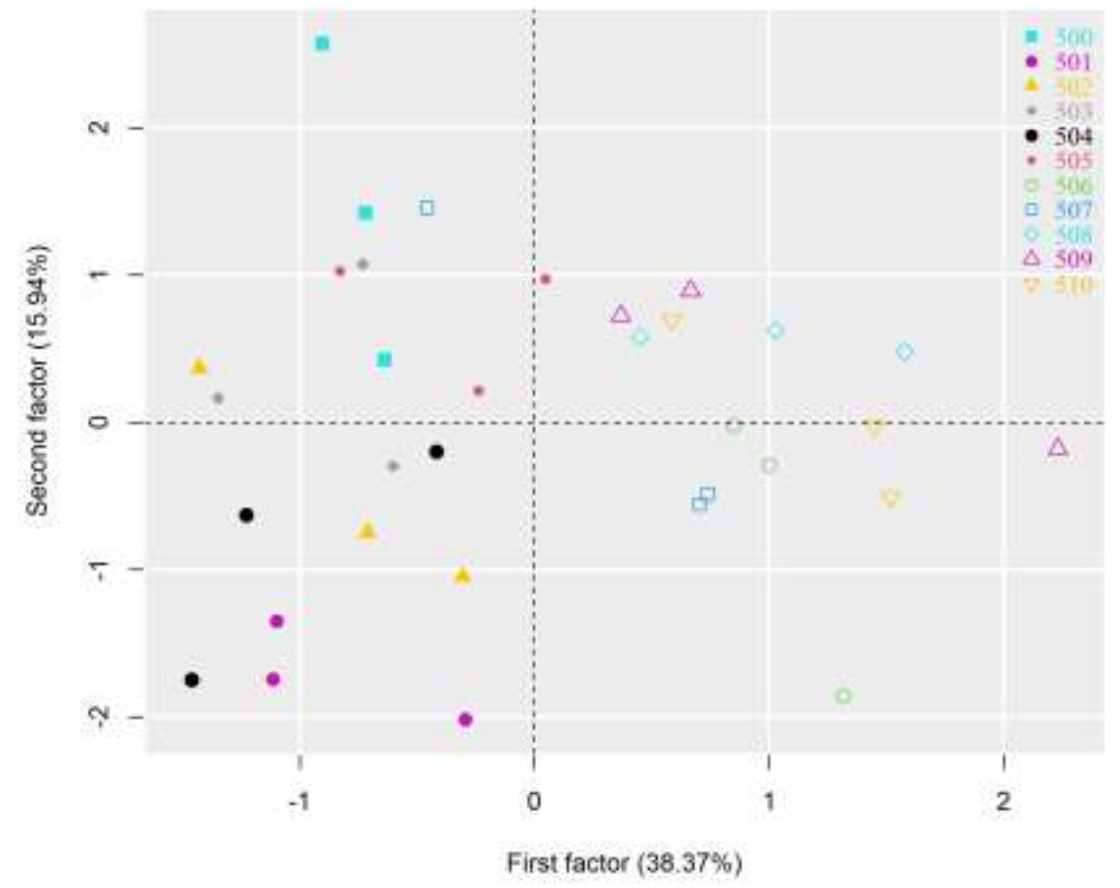

Fonte: Autores.

\subsection{Teste Scott-Knott}

A análise de múltiplos fatores complementam as informações apresentadas pela análise univariada através do teste Scott-Knott (Tabelas 5, 6 e 7), podendo ser uma ferramenta para auxiliar os melhoristas em estratégia de seleção de genótipos, visando o sucesso no programa de melhoramento.

Diante os resultados apresentados nos testes de médias pode se observar que os híbridos experimentais 507 e 509 , apresentam características similares entre si, para a maioria das variáveis avaliadas (alta produtividade, baixa porcentagem de pendoamento, bom vigor de plantas, média incidência de queima de folhas, porcentagem média de raízes tuberosas com classificação 1A $(11,45 \pm 0,21 \%), G(4,17 \pm 0,83 \%)$ e descarte $(53,35 \pm 9,97 \%)$, coloração externa das cenouras apresenta tonalidade laranja médio/intenso, com formato das raízes tuberosa razoável para as exigências do mercado e razoável presença de raízes secundárias), fato que corrobora com os dados observados na MFA (Figura 1). A alta similaridade desses híbridos está relacionada com linhagens genitoras, pois ambos os materiais apresentam em comum o parental masculino.

Os híbridos 502 (EX 4098) e 505 (Juliana), apresentam em comum notas para vigor de planta, incidência de queima de folhas, cor externa, presença de ombro verde e a porcentagem de cenouras classificadas como 1A, 2A e descartados. 
Tabela 5. Número total de cenouras (NTC), produtividade total (Prod), porcentagem de pendoamento (Pend), vigor de planta (VP) e incidência de queima de folhas (IQF) em 11 genótipos de cenoura.

\begin{tabular}{cccccc}
\hline Código & NTC & Prod (ton/ha) & Pend (\%) & VP & IQF \\
\hline 500 & $675.833 \mathrm{a}$ & $87,83 \mathrm{a}$ & $0,00 \mathrm{a}$ & $4,16 \mathrm{a}$ & $4,17 \mathrm{a}$ \\
501 & $588.333 \mathrm{~b}$ & $76,51 \mathrm{~b}$ & $0,00 \mathrm{a}$ & $3,50 \mathrm{a}$ & $2,33 \mathrm{a}$ \\
502 & $650.000 \mathrm{a}$ & $72,34 \mathrm{~b}$ & $1,29 \mathrm{~b}$ & $3,67 \mathrm{a}$ & $3,33 \mathrm{a}$ \\
503 & $675.000 \mathrm{a}$ & $88,65 \mathrm{a}$ & $0,49 \mathrm{~b}$ & $4,00 \mathrm{a}$ & $3,33 \mathrm{a}$ \\
504 & $629.166 \mathrm{a}$ & $91,08 \mathrm{a}$ & $0,12 \mathrm{a}$ & $3,33 \mathrm{a}$ & $3,17 \mathrm{a}$ \\
505 & $579.166 \mathrm{~b}$ & $88,47 \mathrm{a}$ & $0,00 \mathrm{a}$ & $3,83 \mathrm{a}$ & $3,33 \mathrm{a}$ \\
506 & $540.000 \mathrm{~b}$ & $66,51 \mathrm{~b}$ & $4,08 \mathrm{c}$ & $3,67 \mathrm{a}$ & $2,83 \mathrm{a}$ \\
507 & $655.000 \mathrm{a}$ & $83,26 \mathrm{a}$ & $0,66 \mathrm{~b}$ & $3,83 \mathrm{a}$ & $3,00 \mathrm{a}$ \\
508 & $556.666 \mathrm{~b}$ & $73,13 \mathrm{~b}$ & $0,45 \mathrm{~b}$ & $4,33 \mathrm{a}$ & $3,00 \mathrm{a}$ \\
509 & $520.833 \mathrm{~b}$ & $79,45 \mathrm{a}$ & $0,79 \mathrm{~b}$ & $4,33 \mathrm{a}$ & $3,33 \mathrm{a}$ \\
510 & $547.500 \mathrm{~b}$ & $66,70 \mathrm{~b}$ & $1,15 \mathrm{~b}$ & $4,00 \mathrm{a}$ & $3,17 \mathrm{a}$ \\
\hline CV $(\%)$ & 9,50 & 9,39 & 19,73 & 9,60 & 10,91 \\
\hline
\end{tabular}

Médias seguidas das mesmas letras na coluna não diferem entre si segundo o teste de Scott \& Knott a 5\% de probabilidade. Fonte: Autores.

Tabela 6. Porcentagem de cenouras classificadas como descartes, 1A, 2A e G em 11 genótipos de cenoura.

\begin{tabular}{|c|c|c|c|c|}
\hline Código & Descarte & $\mathbf{1 A}$ & $2 \mathrm{~A}$ & $\mathbf{G}$ \\
\hline 500 & $38,60 \mathrm{a}$ & $16,44 \mathrm{a}$ & $35,07 \mathrm{a}$ & $9,88 \mathrm{a}$ \\
\hline 501 & $34,35 \mathrm{a}$ & $20,11 \mathrm{a}$ & $41,07 \mathrm{a}$ & $4,45 \mathrm{a}$ \\
\hline 502 & $41,98 \mathrm{a}$ & $20,37 \mathrm{a}$ & $33,34 \mathrm{a}$ & $4,30 \mathrm{a}$ \\
\hline 503 & $45,06 \mathrm{a}$ & $22,21 \mathrm{a}$ & $25,15 \mathrm{a}$ & $7,55 \mathrm{a}$ \\
\hline 504 & $45,29 \mathrm{a}$ & $14,29 \mathrm{a}$ & $36,73 \mathrm{a}$ & $3,68 \mathrm{a}$ \\
\hline 505 & $40,01 \mathrm{a}$ & $17,05 \mathrm{a}$ & $31,76 \mathrm{a}$ & $11,18 \mathrm{a}$ \\
\hline 506 & $55,48 \mathrm{a}$ & $14,01 \mathrm{a}$ & $26,12 \mathrm{a}$ & $4,37 \mathrm{a}$ \\
\hline 507 & $46,30 \mathrm{a}$ & $11,60 \mathrm{a}$ & $37,91 \mathrm{a}$ & $4,17 \mathrm{a}$ \\
\hline 508 & $54,58 \mathrm{a}$ & $14,57 \mathrm{a}$ & $25,46 \mathrm{a}$ & $5,39 \mathrm{a}$ \\
\hline 509 & $60,41 \mathrm{a}$ & $11,31 \mathrm{a}$ & $25,27 \mathrm{a}$ & $2,99 \mathrm{a}$ \\
\hline 510 & $57,53 \mathrm{a}$ & 13,77 a & $26,38 \mathrm{a}$ & $2,31 \mathrm{a}$ \\
\hline $\mathrm{CV}(\%)$ & 22,33 & 33,3 & 30,48 & 76,38 \\
\hline
\end{tabular}

Médias seguidas das mesmas letras na coluna não diferem entre si segundo o teste de Scott \& Knott a 5\% de probabilidade. Fonte: Autores.

Ainda de acordo com o teste de médias os genótipos 500 (SV1099DT) e 506 (Brazlândia) apresentam em comum bom vigor de planta, uma média de 15,22 $\pm 1,71 \%$ de raízes classificadas como $1 \mathrm{~A}$, pouca presença de raízes secundárias e pouca presença de ombro verde. É importante salientar que a cenoura cultivar Brazlândia apresenta polinização aberta, e assim como outras cultivares (Alvorada, BRS Planalto, Brasília Calibrada Média, Brasília RL, Carandaí, Suprema, dentre outras) são derivadas a partir da cv. Brasília (lançada em 1981), na qual várias empresas de hortaliças desenvolveram programas de melhoramento genético a partir desse material, devido a sua grande variabilidade genética, os trabalhos de seleção apresentaram ganhos expressivos, fazendo com que as cultivares derivadas de Brasília sejam bastante distintas morfologicamente da cultivar original (Grangeiro, et al., 2012; Carvalho \& Silva, 2017). 
Tabela 7. Nota para coloração externa da cenoura (CE), coloração do córtex (CC), coloração do núcleo central (NC), forma da cenoura, presença de raízes secundárias (PR) e ombro verde (OV) em 11 genótipos de cenoura.

\begin{tabular}{ccccccc}
\hline Código & $\mathbf{C E}$ & $\mathbf{C C}$ & $\mathbf{N C}$ & Forma & PR & OV \\
\hline 500 & $3,66 \mathrm{c}$ & $3,66 \mathrm{~b}$ & $3,66 \mathrm{a}$ & $3,96 \mathrm{~b}$ & $3,53 \mathrm{~b}$ & $4,40 \mathrm{a}$ \\
501 & $5,00 \mathrm{a}$ & $3,96 \mathrm{a}$ & $3,70 \mathrm{a}$ & $3,50 \mathrm{c}$ & $3,53 \mathrm{~b}$ & $4,40 \mathrm{a}$ \\
502 & $3,93 \mathrm{c}$ & $3,96 \mathrm{a}$ & $3,96 \mathrm{a}$ & $4,43 \mathrm{a}$ & $4,00 \mathrm{a}$ & $3,76 \mathrm{~b}$ \\
503 & $3,76 \mathrm{c}$ & $4,23 \mathrm{a}$ & $4,33 \mathrm{a}$ & $3,90 \mathrm{~b}$ & $3,53 \mathrm{~b}$ & $4,17 \mathrm{a}$ \\
504 & $4,23 \mathrm{~b}$ & $4,10 \mathrm{a}$ & $3,76 \mathrm{a}$ & $4,16 \mathrm{a}$ & $4,23 \mathrm{a}$ & $3,66 \mathrm{~b}$ \\
505 & $3,50 \mathrm{~d}$ & $3,60 \mathrm{~b}$ & $3,40 \mathrm{a}$ & $3,90 \mathrm{~b}$ & $3,63 \mathrm{~b}$ & $3,83 \mathrm{~b}$ \\
506 & $3,56 \mathrm{~d}$ & $3,50 \mathrm{c}$ & $2,96 \mathrm{~b}$ & $3,43 \mathrm{c}$ & $3,26 \mathrm{~b}$ & $4,10 \mathrm{a}$ \\
507 & $3,53 \mathrm{~d}$ & $3,36 \mathrm{c}$ & $3,13 \mathrm{~b}$ & $3,43 \mathrm{c}$ & $3,06 \mathrm{~b}$ & $4,06 \mathrm{a}$ \\
508 & $3,33 \mathrm{~d}$ & $3,13 \mathrm{~d}$ & $2,63 \mathrm{~b}$ & $3,63 \mathrm{c}$ & $3,23 \mathrm{~b}$ & $3,76 \mathrm{~b}$ \\
509 & $3,46 \mathrm{~d}$ & $3,10 \mathrm{~d}$ & $2,60 \mathrm{~b}$ & $3,40 \mathrm{c}$ & $3,66 \mathrm{~b}$ & $3,80 \mathrm{~b}$ \\
510 & $3,36 \mathrm{~d}$ & $2,93 \mathrm{~d}$ & $2,56 \mathrm{~b}$ & $3,73 \mathrm{c}$ & $3,53 \mathrm{~b}$ & $3,63 \mathrm{~b}$ \\
\hline CV $(\%)$ & 5,11 & 5,33 & 9,30 & 5,09 & 7,33 & 8,34 \\
\hline
\end{tabular}

Médias seguidas das mesmas letras na coluna não diferem entre si segundo o teste de Scott \& Knott a 5\% de probabilidade. Fonte: Autores.

Já o híbrido comercial 503 (AGR-123) apresenta características que assemelham ao híbrido experimental 510, como bom vigor de plantas, média incidência de queima de folhas, quantidade razoável/pouca de raízes secundárias, além de uma média de $25,76 \pm 0,86 \%$ de raízes classificadas como $2 \mathrm{~A}$.

O teste de Scott e Knott (análise univariada) permite a seleção de materiais promissores, por meio da comparação de médias, mas não é possível uma visualização explícita de grupos divergentes, entretanto, o uso de ambas as análises, univariada e multivariada, facilita a obtenção de maiores informações sobre os genótipos estudados. A associação dessas técnicas tem sido utilizada em programas de melhoramento genético de diversas culturas, sem estabelecer uma conexão crítica entre elas (Araújo, et al., 2016; Grigolo et al., 2018).

De maneira geral, os resultados apresentados revelam baixa diversidade genética entre os onze genótipos de cenoura avaliados nessa pesquisa, fato também observado em outros estudos com diversidade genética em populações de cenoura. Segundo Maksylewicz e Baranski (2013) e Carvalho e Silva (2017) a baixa diversidade genética entre populações, pode estar atribuída ao modo de reprodução da cenoura, pois espécies alógamas são mais capazes de preservarem a diversidade genética, se comparado a espécies autógamas. Pois o sistema de reprodução favorece a ocorrência de fluxo gênico entre as populações, aumentando a diversidade genética dentro das populações e diminuindo a diferenciação genética entre elas (Zanettini \& Cavalli, 2003).

Assim, deve-se evitar a hibridação entre genótipos do mesmo padrão de similaridade, visando maximizar a variabilidade indispensável em qualquer programa de melhoramento. Entretanto, para a escolha dos genitores deve-se atentar não apenas para a divergência genética, mas também para o desempenho superior nas principais características agronômicas da cultura, tais como caracteres produtivos e qualitativos (Cruz et al., 1994). O conhecimento da dissimilaridade e similaridade pode contribuir para o planejamento racional do melhoramento genético, otimizar o manejo dos acessos do banco de germoplasma e favorecer o conhecimento dos padrões de variação fenotípica e a divergência das espécies. Essas informações poderão ser úteis nas próximas etapas do programa de melhoramento genético de cenoura de verão, visando o desenvolvimento de híbridos a partir de combinações entre as populações com maior divergência genética entre si e que apresentem boas característica das raízes tuberosas. 


\section{Conclusão}

1) A técnica de análise de múltiplos fatores (MFA) permite estabelecer as similaridades entre os genótipos estudados, revelando que 36,36 \% dos genótipos comerciais (501, 503 e 505) e experimentais (510) apresentam alta similaridade entre si. Isto mostra que o background destes materiais tem a mesma origem;

2) A análise de fatorial exploratória estabeleceu as correlações das variáveis, e possibilitou a projeção dos pontos que formam os genótipos ora estudados, uma separação entre os genótipos comerciais e experimentais;

3) As especificidades apresentadas pelos genótipos foram explicadas no teste de média Scott-Knott, permitindo relacionar os genótipos pelos agrupamentos das variáveis estudadas, tais como 507 e 509; 502 (EX 4098) e 505 (Juliana); 500 (SV1099DT) e 506 (Brazlândia); e 503 (AGR-123) e 510.

4) Por se tratar de descritores agronômicos e morfológicos, altamente influenciáveis por fator ambiental o ensaio deve ser repetido em diferentes ambientes.

\section{Referências}

Abdi, H., Williams, L., \& Valentin, D. (2013). Multiple factor analysis: principal component analysis for multitable and multiblock data sets. WIREs Computational Statatistics, 5 (2), 149-179. 10.1002/wics.1246

Araújo, J. C., Telhado, S. F. P., Sakai, R. H., Ledo, C. A. S., \& Melo, P. C. T. (2016). Univariate and multivariate procedures for agronomic evaluation of organically grown tomato cultivars. Horticultura Brasileira 34: 374-380, 2016. 10.1590/S0102-05362016003011

Buratto, J. S., Santos Neto, J., \& Moda-Cirino, V. (2016). Desempenho agronômico e dissimilaridade genética entre acessos de amendoim por variáveis multicategóricas. Scientia Agraria Paranaensis, 15(3), 324-331. 10.18188/sap.v15i3.13125

Carvalho, A. D. F., \& Silva, G. O. (2017). Genetic divergence among carrot genotypes through agronomic characteristics. Revista Agro@mbiente, 11 (2), 137144. 10.18227/1982-8470ragro.v11i2.3642

Cecon, P. R., Silva, F. F. E., Ferreira, A., Ferrão, R. G., Carneiro, A. P. S., Detmann, E., Faria, P.N., \& Morais, T. S. S. (2008). Análise de medidas repetidas na avaliação de clones de café 'Conilon'. Pesquisa Agropecuária Brasileira, 43 (1), 1171-1176. 10.1590/S0100-204X2008000900011

Cruz, C. D., Carvalho, S. P., \& Vencovsky, R. (1994). Estudos sobre a divergência genética II. Eficiência da predição do comportamento de híbridos com base na divergência de progenitores. Revista Ceres, v. 41(1) 183-194. http://www.ceres.ufv.br/ojs/index.php/ceres/article/view/2070

Cruz, C. D., Regazzi, A. J., \& Carneiro, P. C. S. (2012). Modelos biométricos aplicados ao melhoramento genético. Ed. da UFV, 514p.

Escofier, B., \& Pagès, J. (2008). Analyses factorielles simples et multiples: objectifs, méthodes et interprétation. (4a ed.), Dunod, 318 p.

FAOSTAT - Food and Agriculture Data. Produtividade da Cenoura (2017). http://www.fao.Org/faostat/en/\#data/QC

Ferreira, E. B., Cavalcanti, P. P., \& Nogueira D. A. (2021). ExpDes: Pacote Experimental Designs. URL <https://cran.rstudio.com/web/packa ges/ExpDes/index.html>. R package version 1.2.1.

Grangeiro, L. C., Azevêdo, P. E., Nunes, G. H. S., Dantas, M. S. M., \& Cruz, C. A. (2012). Desempenho e divergência genética de cenoura 'Brasília' em função da procedência das sementes. Horticultura Brasileira, 30 (1), 137-142. 10.1590/S0102-05362012000100023

Grigolo, S., Fioreze, A. C. C. L., Denardi, S. \& Vacari, J. (2018). Implicações da análise univariada e multivariada na dissimilaridade de acessos de feijão comum. Revista de Ciências Agroveterinárias 17(3), 351-360. https://www.revistas.udesc.br/index.php/agroveterinaria/article/view/9324

Hiranvarachat, B., \& Devahastin, S. (2014). Enhancement of microwave-assisted extraction via intermittent radiation: Extraction of carotenoids from carrot peels. Journal of Food Engineering, 126 (1), 17-26, 2014. 10.1016/j.jfoodeng.2013.10.024

Jelihovschi, E., Faria, J. C., \& Allaman, I. B. (2014). Scott-Knott: A Package for Performing the Scott-Knott Clustering Algorithm in R. Trends in Computational and Applied Mathematics, 15 (1), 3-17. 10.5540/tema.2014.015.01.0003

Maksylewicz, A., \& Baranski, R. (2013) Intra-population genetic diversity of cultivated carrot (Daucus carota L.) assessed by analysis of microsatellite markers. Acta Biochimica Polonica, 60 (1) 753-760. https://pubmed.ncbi.nlm.nih.gov/24432327/

Nick, C., \& Borém, A. (2016). Cenoura: do plantio à colheita. Editora UFV. 179 p.

Oliveira, C. D., Braz, L. T. E., \& Banzatto, D. A. (2005). Adaptabilidade e estabilidade fenotípica de genótipos de cenoura. Horticultura Brasileira, 23 (3) 743-748. 10.1590/S0102-05362005000300011

Ossani, P. C., \& Cirillo, M. A. (2021). MVar: Multivariate Analysis. URL <https://cran.r-project.org/web/packages/MVar/index.html>. R package version 2.1.7.

Rencher, A. C., \& Christensen, W. F. (2012). Methods of Multivariate Analysis. (3a ed.), J. Wiley, 758 p. 
Research, Society and Development, v. 10, n. 13, e173101321145, 2021

(CC BY 4.0) | ISSN 2525-3409 | DOI: http://dx.doi.org/10.33448/rsd-v10i13.21145

R Development Core Team. (2021) R: A language and environment for statistical computing. R Foundation for Statistical Computing. Vienna: Vienna University of Economics and Business. http://www.R-project.org

Scott, A. J., \& Knott, M. (1974). A Cluster Analysis Method for Grouping Means in the Analysis of Variance. Biometrics, 30 (3), 507512. https://www.jstor.org/stable/2529204

Souza, D. C., Ossani, P. C., Vilela, L. V., Cirillo, M. Â., Silva, L. F. L. S., \& Xavier, J. B. (2019). Variabilidade genética entre cultivares comerciais e híbridos experimentais de morangueiro com enfase em análise de múltiplos fatores. Magistra, 30: 48-59. https://magistraonline.ufrb.edu.br/index.php/magistra/article/view/725

Zanettini, M. H. B., \& Cavalli, S. S. Variabilidade genética em função do modo de reprodução. In: Freitas LB, Bered F, editors. Genética e evolução vegetal.: Editora UFRGS; 2003. p.177-187. 